Voix et Images

voixetimages

\title{
Entrevue avec France Théoret
}

\section{Patricia Smart}

Volume 14, numéro 1 (39), automne 1988

France Théoret : narratrice de la subjectivité

URI : https://id.erudit.org/iderudit/200748ar

DOI : https://doi.org/10.7202/200748ar

Aller au sommaire du numéro

\section{Éditeur(s)}

Université du Québec à Montréal

\section{ISSN}

0318-9201 (imprimé)

1705-933X (numérique)

Découvrir la revue

\section{Citer ce document}

Smart, P. (1988). Entrevue avec France Théoret. Voix et Images, 14(1), 11-23.

https://doi.org/10.7202/200748ar d'utilisation que vous pouvez consulter en ligne.

https://apropos.erudit.org/fr/usagers/politique-dutilisation/ 


\title{
Entrevue avec France Théoret
}

\author{
par Patricia Smart, université Carleton, Ottawa
}

V. \& I. - La voix qui parle dans vos écrits est toujours autobiographique, mais elle déborde du personnel pour rejoindre le collectif, le fictif. Pourriez-vous préciser comment vous envisagez ce lien entre le personnel et le collectif dans votre écriture?

F. Théoret - Je narre la subjectivité en liaison avec une réalité donnée, je la narre dans ce qu'elle possède de singulier et dans ce qu'elle a de réducteur et d'excentrique. Je sais que je ne peux arracher une part importante qui me constitue: le fait d'être femme, née dans un milieu précis, au Québec. C'est ce qu'on appelle les racines. Mais je ne cherche pas à m'enraciner, j'emploie le mot excentrer depuis les tout premiers textes pour marquer la nécessité d'agiter les coordonnées, d'aller vers l'extériorité. Ces coordonnées font partie de ma mémoire. Si la voix est autobiographique, seuls quelques mots remémorés suffisent pour écrire.

V. \& I. - Je voudrais commencer par vous poser quelques questions sur votre vie. Où êtes-vous née?

F. Théoret - Je suis née à Montréal, en pleine urbanité, dans le quartier Maisonneuve. J'ai grandi en ville. Après la guerre, mon père a acquis un premier commerce. Quand j'ai eu 8 ans, la famille a emménagé sur les lieux du restaurant-épicerie comme on disait à l'époque. Mis à part le fait que ma mère y travaillait et que nous apportions notre contribution au commerce, notre vie pouvait ressembler à celle de la majorité. Les années cinquante sont demeurées dans ma mémoire une décennie assez morne où le conformisme social, moral et intellectuel était prégnant jusque dans les détails, pas seulement dans la famille, mais aussi à l'école. Ainsi, j'ai vécu dans un milieu où la vie était organisée, à la minute près, sauf durant les vacances.

Le petit commerçant possède son autonomie, il n'est cependant pas à l'abri de la faillite. Dans ma fiction, j'ai déjà abordé le thème de l'argent, ce qui laissait présumer que j'ai grandi dans un milieu pauvre. Pourquoi? l'argent est une problématique importante et touche ce qui entretient le consensus social, le conformisme. On est plus brutal ou plus sophistiqué à propos de la question économique selon son appartenance sociale. Dans le commerce, j'ai été confrontée à ce qui peut demeurer une relative abstraction quand on est enfant. L'argent médiatise bien des valeurs, j'ai compris cela très tôt. 
V. \& I. - Vous parlez de la campagne aussi bien que de la ville quand vous évoquez l'enfance. Est-ce que vous avez vécu à la campagne?

F. Théoret - Mes parents ont déménagé à la campagne, je venais d'avoir 15 ans. Je n'ai pas passé une année entière à Saint-Colomban. J'y allais les fins de semaines et durant les vacances, je continuais à travailler au commerce. J'ai eu un choc. Ayant grandi dans un milieu organisé, je considérais essentiel de pouvoir choisir mes amies, par exemple.

V. \& I. - Ce qui n'existait pas à la campagne...

F. Théoret - Il y avait trop peu de familles à Saint-Colomban, et le niveau de vie était réellement bas, pas pour ma famille encore une fois. $\grave{A}$ la ville, un certain anonymat nous protège; $j$ 'ai vu dans ce village des choses que je n'ai jamais vues à la ville.

V.\& I. - Comme...

F. Théoret - Rencontrer, un jour, une femme qui a été échangée contre une vache. Elle n'habitait pas Saint-Colomban, elle passait par là. Voir des enfants mangeant des patates qu'ils écrasaient sur la table. Au village, j'ai connu un fort sentiment de régression.

V. \& I. - Dans votre enfance, est-ce que vous saviez que vous vouliez devenir écrivaine? Quand avez-vous su cela?

F. Théoret - L'idée m'est venue à l'âge de 11 ans. Une amie recevait le journal François. Chaque mois, en page frontispice, il y avait la photo d'un enfant qui nommait ce qu'il désirait devenir. Sous la photo d'un garçon, un mot: écrivain. Mon amie dit qu'elle sera écrivain; je dis: moi aussi. J'empruntais des livres à la bibliothèque de l'école et j'entretenais une correspondance qui allait prendre des dimensions importantes.C'est grâce à l'école et à la correspondance que j'ai découvert que j'aimais écrire. À 14 ans, j'adorais la narration et je m'imaginais romancière.

\section{V. \& I. - Qu'est-ce que vous lisiez à cette époque?}

F. Théoret - Je lisais Pêcheur d'Islande de Pierre Loti, les romans édifiants de Frison-Roche et des romans populaires. Ces romans racontaient la même histoire, celle de deux jeunes filles: l'une est belle, l'autre pas; l'une est aimable, l'autre détestable; l'une réussit, l'autre est sacrifiée.

\section{V. \& I. - Et ça mène au mariage...}

F. Théoret - Oui. Tous ces livres se terminent de la même manière, par le mariage de l'une, tandis que l'autre n'a pas droit au bonheur. Ma soif de lecture n'était jamais assouvie, je lisais n'importe quoi. La bibliothèque des enfants ne 
m'intéressait plus, il fallait avoir 16 ans pour avoir accès à la bibliothèque des adultes. J'ai tenté d'avoir une carte malgré ma timidité excessive. Et il n'y avait pas de librairie où j'habitais. Un long été à la ville, Graziella de Lamartine et les Rêveries du promeneur solitaire de Rousseau évoquaient mille promesses.

\section{V. \& I. - Est-ce que c'étaient les religieuses qui vous en avaient parlé?}

F. Théoret - Les citations du livre de français étaient accompagnées du titre de l'cuvre et du nom de l'auteur. Pendant un long moment de mon adolescence, j'ai eu le sentiment de régresser parce que je ne pouvais pas lire ce qui excitait mon imagination.

Ainsi en a-t-il été du désir d'écrire. Je poursuivais mes correspondances, mais $\mathrm{j}$ 'avais le sentiment de toumer en rond parce que je ne lisais pas.

V. \& I. - Mais votre rapport à la langue doit remonter plus loin, jusqu'à la petite enfance.

F. Théoret - Je rêvais à la langue. Je me promenais avec des mots, je m'endormais avec des mots quand j'avais 5 ou 6 ans. J'ai déjà parlé de la difficulté de parole, la critique a conclu aux difficultés d'apprentissage. Ce n'était pas le cas. Mais puisque j'ai donné prise à cette affirmation, je dois m'expliquer.

Pour avoir longtemps joué avec les mots, je me rappelle mon apprentissage. Cet apprentissage est présent, alors qu'il aurait dû être oublié au fur et à mesure que ma compétence linguistique se modifiait. Dans mon milieu, on travestissait beaucoup les mots. Je vais donner des exemples. On disait: médi, ménuit, pélulle, castonnade; on parlait de varmine ou encore de cartron; on disait drabe pour beige, canard pour bouilloire. La liste pourrait s'allonger. Avant d'aller à l'école, je tentais différents usages. À l'école, j'apprends l'orthographe, ma langue change et je suis consciente assez tôt que je deviens différente. Il en a été ainsi pour la syntaxe. Je tentais d'intégrer à l'oral ce que j'apprenais dans l'écrit. J'étais consciente d'être à l'écart par rapport à mon milieu. Non seulement je n'en jouissais pas, mais cet écart me rendait timide, d'où certains malaises à prendre la parole et non pas avec l'écrit. Toujours en faisant mes études, j'ai eu conscience que je précisais ma langue.

V. \& I. - Oui, mais ça va dans les deux sens finalement. II a fallu que vous maîtrisiez la langue, mais en même temps, vous n'avez pas accepté de laisser la réalité en arrière. Pourrait-on dire que vous voulez travailler la langue jusqu'à ce que la voix de votre mère et sa façon de parler parlent à travers le langage écrit?

F. Théoret - Non, car ce n'est pas du mimétisme. Mais effectivement, je n'ai pas oublié le parcours. Je ne sais toujours pas pourquoi mes mots n'ont pas oublié le parcours. La voix autobiographique parle la langue de maintenant et se rappelle celle des origines, donc celle de la mère. Et ce langage de ma mère subit une tranformation, une métamorphose. 
V. \& I. - Vous dites dans Entre raison et déraison qu'on étudie et qu'on lit "en cachette» au Québec, que dans votre enfance, quand on vous surprenait en train de lire, on disait que vous «tiriez du grand». Est-ce que ce manque d'encouragement a influencé votre rapport à l'écriture? Je pense aussi à la jeune Louise Aubert, dans «L'homme qui peignait Staline», à qui on donnait le nom de «Marquise», quand elle était enfant, à cause de son goût pour les belles choses. Vos personnages portent les cicatrices d'un milieu qui interdit le moindre signe d'individualité ou de différence...

F. Théoret - Le livre n'était pas valorisé, il n'y avait pas de livre à la maison. Ça n'a pas vraiment influencé mon rapport à la lecture puisque je lisais quand même. Dans ma fiction, je décris certains milieux rébarbatifs au livre et à la culture parce que les valeurs sont ailleurs. Quand la lecture et la culture n'ont pas d'importance, elles peuvent être un sujet de risée. Cette attitude d'indifférence n'interdit pas nécessairement toute manifestation d'individualité.

Il y a eu un moment durant mon adolescence où j'ai été profondément religieuse. Alors la littérature était marquée du signe de l'absolu. Évidemment. On n'est jamais digne de l'absolu. J'écrivais des poèmes, des débuts de récit que je déchirais. Aller au bout d'une démarche relevait du défi.

V. \& I. - Parmi vos plus beaux portraits littéraires, il y a des adolescentes comme Louise Valois, dans Nous parlerons comme on écrit, comme l'adolescente dans «Ceci n'est pas un lac». L'adolescence semble un moment de vérité particulière pour vous.

F. Théoret - L'adolescence est un âge fort complexe qui peut poser toutes les questions en même temps. Des questions d'orientation personnelle, sexuelle, sociale. Louise Valois, par exemple, fait face à la question sexuelle à cause de sa mère qui fait une fausse couche. L'adolescente ignorait que sa mère était enceinte et tout de suite après la fausse couche, la mère veut lui montrer le fotus. La fille refuse et elle panique.

L'adolescente dans «Ceci n'est pas un lac» est seule. Elle en profite pour formuler ses questions, entre autres, à propos du temps. Elle en arrive à la conclusion que le temps la constitue. Elle dit: si je tue le temps, je me tue. Ses doutes lui viennent de son opposition à la mère qui répète qu'on ne connaît pas l'avenir et qu'à chaque jour suffit sa peine. À cet âge, on est totalement soimême et totalement le reflet de son milieu.

V. \& I. - L'adolescence pour les filles dans vos écrits, c'est aussi la découverte presque insoutenable de ce qu'il y a d'intolérable dans la réalité que vivent leurs mères. Dans votre récit «Onze ans», il y a un portrait extraordinaire de la mère et de sa fille de onze ans qui veut sauver le «charme indéfinissable» de sa mère contre tout ce qui le détruit. On dirait que cette souffrance des mères, qui remonte très loin, est véritablement le moteur principal de votre écriture... 
F. Théoret - Dans Une voix pour Odile, je parlais de la souffrance des mères qui remonte très loin. Le personnage de la mère revient dans les récits que j'écris actuellement. J'écris sur la mère en parlant de la répétition sous divers angles de perception. Quand on a conscience de la répétition, une souffrance demeure inexprimée, les mères que j'imagine ont conscience d'une forme de répétition qui n'est pas très éloignée de la fatalité.

V. \& I. - Est-ce que c'est pendant l'adolescence que vous avez découvert la musique aussi?

F.Théoret - Pas la musique classique. Mais la musique religieuse, les chansons populaires, celles d'Edith Piaf, par exemple. Plus tard, les chansonniers québécois et français. Rien de systématique à vrai dire.

V. \& I. - Il y a le beau passage dans Nous parlerons comme on écrit quand la narratrice pratique sa voix, projette sa voix de l'intérieur. C'est comme si la musique, la voix réunissaient toutes les dimensions de l'existence.

F. Théoret - Dans la trentaine, j'ai découvert la magie de la musique, de la musique vocale en particulier. J'ai osé décrire six musiques vocales que j'écoutais continuellement en écrivant la première partie de Nous parlerons comme on écrit. De Purcell à Luigi Nono, passant par Liszt, Schönberg, Mahler et Varèse. La musique m'invitait à la fuite, pour ne conserver qu'un seul désir: inscrire ma démarche littéraire. Je ne crois pas qu'on puisse écrire rien de valable à partir de ce qui fait corps avec le langage si on ne garde pas un rythme, un ton particulier, une écriture qui n'a finalement rien à voir avec le langage parlé.

V. \& I. - Vos écrits sont féministes, tout le monde le remarque, mais ils sont aussi très québécois. Il me semble que vous avez un rapport très conscient avec le milieu québécois et avec vous-même en tant que Québécoise. Est-ce que c'est un rapport problématique?

F. Théoret - Oui, certainement un rapport problématique. J'écris parfois en pensant au Québec. Il arrive que je m'inclus en parlant des Québécois, à cause de la violence de certaines affirmations.

Le rapport que nous établissons ici avec la connaissance, la lecture et la culture n'est jamais prioritaire et, à cause de cela, demeure précaire. On ne peut pas légitimement consacrer sa vie à l'écriture, à la connaissance, à la lecture. Il faut en imposer le fait et en supporter les conséquences. Je ne pense pas que la différence devrait constituer une marginalité, elle est plutôt un aspect possible dans la collectivité. Un malaise profond provient de l'absence de légitimité. À ce sujet, je vais vous raconter une anecdote qui n'a rien de bien singulier.

Il y a trois ou quatre ans, au début d'une session, j'ai dit à un groupe d'étudiants qu'ils devraient débourser environ $15 \$$ pour les livres dont ils auraient besoin. Habituellement, je prenais des précautions oratoires; il y avait 
quand même un murmure qui suivait l'annonce. Cette fois-là, j'ai oublié d'en prendre. Les étudiants ont réagi négativement et au lieu de tenter de les raisonner, j'ai parlé spontanément du relatif confort dans lequel ils vivent et de la valeur que constitue une bibliothèque. Ils ont fait bloc contre moi quand $j$ 'ai dit que dans des pays d'Europe de l'Est, par exemple, moins riches économiquement, on accordait une valeur au fait de posséder des livres. Leur réaction m'a prouvé une fois de plus que le rapport aux livres fait problème chez nous.

V. \& I. - En vous lisant, on ne peut pas s'empêcher de remarquer une certaine noirceur. Ce n'est pas une réalité facile que vous mettez en scène.

F. Théoret - Quand j'écris, je ne cherche pas à m'évader. Jusqu'ici, j'ai souvent écrit en état d'urgence. Le fait d'écrire lentement et de vouloir condenser des problématiques existentielles, une conception du langage, des inventions formelles m'a amenée à multiplier des effets dont je n'étais pas toujours consciente. Quand les mots supportent un certain poids de réel, il y a comme vous dites une certaine noirceur. Jusqu'à maintenant, j'ai souvent écrit contre le temps. Maintenant, j'aimerais écrire avec le temps.

Puis, j'ai écrit sur ce qui rétrécit, diminue les énergies vitales et à propos de la régression qui peut, bien sûr, être un moment de recul pour mieux recommencer, mais qui est tout aussi souvent un moment où l'on expérimente l'abjection.

V. \& I. - Cette colère et cette gêne dont vous parlez à propos des Québécois, il est certain que beaucoup de femmes aussi les ressentent, devant une parole et une connaissance d'où elles ont été exclues. Dans votre essai Entre raison et déraison, vous évoquez le cheminement très long, très pénible que doivent emprunter les femmes avant d'arriver à la conquête de la raison, de la maîtrise et de leur propre subjectivité. Et il me semble qu'il y a là une redéfinition des termes mêmes de la «mâitrise», de la «raison» et de la «subjectivité» qui a des conséquences énormes pour le savoir tel qu'il est envisagé dans la culture patriarcale. Pourriez-vous vous expliquer un peu là-dessus?

F. Théoret - Si le discours et la connaissance ont été des outils de pouvoir, il est néanmoins nécessaire d'en acquérir la maîtrise, puis une maîtrise de son langage propre. Cette idée de la maîtrise, je n'en fais pas une question polémique. Il y a quelques années, on écrivait que l'écriture au féminin recherchait la nonmaîtrise. À mon sens, l'idée est incomplète et, par là, trompeuse. Rechercher la non-maîtrise implique une opposition dialectique au discours de maîtrise. Cette idée de non-maîtrise a fait évoluer l'écriture des femmes en nous posant comme autre dans le langage. Mais les femmes ne sont pas essentiellement autre, ce qui est autre advient par ce qu'elles écrivent. Françoise Collin dit dans la Parole métèque: Déterminer préalablement ce qui est authentiquement aféminin» provoquerait un effet de censure sur les femmes. Je suis d'accord. S'approprier la culture, qui est historiquement masculine, et son langage propre exige une maîtrise, un acte d'affirmation. 
La maîtrise passe par un sujet, il y a des écrivaines qui témoignent de la maîtrise à travers leur subjectivité. L'objectivité traditionnelle dit que le moi est haïssable et que le je est nécessairement narcissique. Je pense que la subjectivité est une grande question de la littérature, non pas dans ce qu'elle a de personnel, mais dans ce qu'elle a de partageable, et qu'il est possible d'objectiver. Finalement, je parle d'une maîtrise qui est une autonomie.

V. \& I. - À partir de ce qu'on est.

F. Théoret - Oui, à partir de ce qu'on est. On ne répète que sa propre expérience.

V. \& I. - Vous formulez là une nouvelle idée de la subjectivité, il me semble.

F. Théoret - Je la ressens plus que je ne l'exprime encore.

V. \& I. - Une identité qui ne soit pas construite par la domination.

F. Théoret - Pour acquérir cette «identité», il faut apprendre, désapprendre, réapprendre. La subjectivité n'est pas une donnée déjà là. Ce qui est donné est là pour être déconstruit, reconstruit, je suis constamment dans le mouvement d'un acquis.

V. \& I. - Comme le disait si bien Aragon, rien n'est jamais acquis. Est-ce cela votre vision du monde finalement?

F. Théoret - Ni à l'homme ni à la femme. Il s'agit d'un continuel mouvement de dépassement. L'idée d'un parcours me stimule. Il y a un parcours dans la langue, dans l'écriture, dans l'existence qui m'intéresse continuellement. Transformer les acquis permet le parcours. Et le parcours devient le but, la finalité. Ma vision du monde est une perception, ce n'est pas une conception du monde. Puisque je fais un parcours, je suis partie prenante du monde.

V.\& I. - En même temps, il me semble que vous prenez énormément de risques par rapport à ce que vous appelez la turbulence intérieure, ce domaine proche de la folie, où l'identité et les certitudes sont menacées par les assauts du monde extérieur, que vous faites entrer avec tant de précision dans le langage. Est-ce que vous ne risquez pas d'être étiquetée comme une écrivaine «déprimante» ou «aliénée» par des gens qui ne veulent pas être dérangés par cette réalité?

F. Théoret - C'est vrai qu'on m'a déjà renvoyé l'image d'une écrivaine «déprimante». Mais rien dans ce que j'écris n'est fataliste, bien au contraire; je connais trop bien le fatalisme pour ne pas le débusquer. Mes personnages luttent contre ce qui est latent, béant en elles, contre ce qui rétrécit l'existence. L'identité est une affaire sociale qui est importante, cependant mes personnages n'arrivent pas à s'en contenter. Voyez-vous, je deviens réductrice quand j'essaie de résumer. 
En réalité, à l'époque où j'écrivais Nécessairement putain et Nous parlerons comme on écrit, je connaissais la phrase de Laure qui dit craindre d'être lue par qui ne peut pas être déchiré. Cependant, je ne pouvais pas écrire autrement à cause du temps qui fuyait, surtout à cause de ma conception du langage: les mots supportent un certain poids de réel.

J'ai hérité d'une éducation rigide. Mon éducation religieuse a été rigide, de même que ma formation au collège. On établissait une distinction nette entre le bien et le mal, entre la raison et la folie. Qu'est-ce que la raison? la déraison? Pour une femme en particulier. Il y a tant d'œuvres où des femmes incapables de poursuivre leur action sombrent dans la démence ou la mort: Antigone, Ophélie, Phèdre, Madame Bovary, Nadja. Ces personnages ont beaucoup compté pour moi.

La déraison touche le pré-langage: il faut l'articuler pour le rendre compréhensible. Ce que j'écris dans «La turbulence intérieure» est un état de panique qui peut durer assez longtemps, un état qui mène à douter de sa raison. Beaucoup de femmes $m$ 'ont dit l'avoir vécu... La turbulence intérieure pose la question de la distance entre le moi et le monde.

V. \& I. - Et beaucoup de femmes le vivent encore, je crois.

F. Théoret - Mais on craint les états limites. Je crois qu'à examiner les états limites, on éloigne d'autant plus la déraison. Si cet état limite existe et qu'il peut être nommé, je formule encore ce qui s'appelle la raison puisque je demeure dans le langage articulé.

Ce qui a rapport à la raison et à la déraison me touche, principalement ce qui fait trahir sa subjectivité.

V. \& I. - Vous privilégiez beaucoup l'image de la fille dans vos écrits - la petite fille ou la vieille petite fille. Est-ce une image de la femme dans la société patriarcale?

F. Théoret - Entre femmes dans la trentaine, nous nous interpellions souvent en disant «les filles» puis en disant «les femmes», au fur et à mesure que chacune atteignait la quarantaine! Nous nous appelions ainsi par affection, par provocation, par désir de déviance. Elle m'est restée, cette image provocatrice de la fille.

Quand il s'agit de la petite fille sage, je parle de la passivité. C'est un sujet tabou pour le féminisme. Mais tout ce qui vit possède des énergies, la petite fille sage en a aussi, elle attend de réunir ses forces pour passer à l'acte. Par la petite fille sage, je souhaite rejouer l'image d'une Ophélie qui n'est plus hantée par sa blancheur, par sa virginité et qui trouve en elle-même les ressources contre sa passivité. 
La vieille petite fille vient du personnage de Lily Briscoe dans la Promenande au phare de Virginia Woolf. J'ai appelé vieille petite fille ce personnage de la célibataire qui peint dans sa chambre et qui est fascinée par cette femme chez qui elle habite, le personnage d'une mère qui a de l'ascendant sur chacun et qui sait organiser une maison comme un royaume. J'ai écrit ce texte pour une revue de psychanalyse, je réfléchissais sur la maternité. Je n'ai pas d'enfant.

Mais, il n'y a pas de vieille petite fille dans ma fiction. Lily Briscoe a bien changé. Le personnage de Ninon dans Nous parlerons comme on écrit a une trentaine d'années. Elle n'est désignée ni comme fille ni comme femme. Je dirais qu'il s'agit d'une adulte responsable dans sa vie sociale, donc d'un personnage neutre. Elle va évoluer à cause d'une crise qui passe par le langage. Ninon s'est mise à questionner les mots les plus usuels qui ordonnent les gestes de son existence. Les mots ne coïncident plus, ce qui est grave; elle croit qu'il n'y a jamais eu de coïncidence entre ses paroles et ses gestes. Elle est profondément troublée par ce qui lui arrive. Selon elle, justement, le fait d'avoir l'âge d'une femme aurait dû en faire un être stable, à l'abri d'une crise. Ninon ne vit pas une crise régressive, bien au contraire. Elle est une Lily Briscoe, plus jeune, en train de rompre avec ce qui a précédé. Fille, femme, adulte, peu importe: elle veut assumer son langage et ne peut le faire au neutre, encore moins dans la fascination comme c'était le cas pour Lily Briscoe.

V. \& I. - Vous avez enseigné pendant dix-neuf ans, jusqu'à l'année passée. Comment ce métier vous apparaît-il?

F. Théoret - Enseigner est un acte important. J'ai aimé ce travail pour la part de beauté que comporte l'acte d'enseigner et le rapport avec les étudiants. J'avais une satisfaction réelle lorsque je percevais une évolution chez les étudiants à la fin d'une session. Ça ne se produisait pas toujours.

J'ai beaucoup enseigné les classiques, Edipe-Roi, Dom Juan; des écrivains contemporains, Beckett, Duras, Orwell. Je m'amusais beaucoup dans Bartelby l'écrivain de Melville. À chaque session, je mettais au programme des œuvres québécoises. J'ai đû défendre l'enseignement de la littérature québécoise. Dans certains cégeps, l'enseignement de la littérature québécoise diminue. Les principales objections étaient de deux ordres: la première, la langue des auteurs; la seconde, on pretend qu'il est impossible d'atteindre une vision universelle à partir de notre littérature. Par opposition à la littérature universelle, la littérature québécoise serait mineure, «nombriliste», enfermante. On se refuse à la légitimer au nom des chefs-d'œuvre de la littérature mondiale. La bataille est loin d'être gagnée.

Si des professeurs n'acceptent pas leur propre littérature, ce n'est pas étonnant que dans les familles, on ne valorise pas le livre et que la culture demeure une valeur empruntée. 
V. \& I. - Vous donnez aussi des ateliers d'écriture. Est-ce que ça vous plaît? Comment enseignez-vous à écrire?

F. Théoret - Je fais des ateliers d'écriture pour femmes depuis trois ans maintenant. Je ne suis pas attachée à une institution. Les participantes ont des expériences souvent très riches, elles viennent développer leur capacité d'écrire. Il n'y a qu'une exigence: écrire.

À l'atelier, j'ai le sentiment qu'on désire ma présence comme écrivaine, alors je cherche par tous les moyens à faire évoluer les participantes dans leur écriture et dans leur perception de la littérature. Nous passons beaucoup de temps à analyser les points forts et les points faibles des textes. Cependant, je ne peux pas enseigner à écrire; écrire n'est pas reproduire, mais créer.

L'esprit d'atelier dont parle Claire Lejeune, j'essaie de le mettre en pratique dans le partage entre les participantes et entre elles et moi. Je tente aussi de rendre conscient le travail littéraire et de développer un esprit critique par rapport à son écriture, mais pas un esprit critique qui serait un surmoi destructeur.

J'aime transmettre ce que je sais.

V. \& I. - Dans votre écriture, vous avez touché à tous les genres - poésie, roman, thêâtre, essai.

F. Théoret - Il me fallait découvrir, en pratiquant l'écriture, ma ligne de force, mon courant de fond, ma spécificité littéraire comme chaque écrivain doit le faire. Au départ, j'ai cherché une vision globale de l'écriture. Je croyais l'avoir trouvée au moment où j'ai commencé Une voix pour Odile en 1974. Je me suis vite rendu compte que j'étais en complète contradiction. Je souhaitais rendre ma perception sous toutes ses formes en rendant le «je» irrepérable. En tant que sujet parlant dans le texte, je devais être partout et nulle part. Le «je» pouvait apparaître, disparaître, se camoufler, il serait insaisissable. Ainsi, je pourrais écrire des affirmations, des négations, des dénégations sans $m$ 'y confronter. Puis, tout a basculé sous l'influence de l'écriture des femmes. La question du sujet est devenue primordiale. Les fragments d'Une voix pour Odile déploient différents moments de la voix.

Si je suis poète, je le suis dans la prose. Et il y a une ambiguïté dans la poésie en prose. Il se peut qu'un jour, on me convainque du contraire. Je ne demande pas mieux! Je suis en train de dire que ma pratique de la poésie en prose est ambiguẽ et pourtant, je sais que je désire la poursuivre. Si je n'arrive pas à la justifier, disons de façon pleinement significative au niveau formel, la poésie en prose permet une exposition de la voix du sujet tel que le roman ne le permet pas, à quelques exceptions près. Je pense aux romans de Carole Massé et de Louise Bouchard. En partie aussi à Nous parlerons comme on écrit.

Mais le roman m'intéresse dans la mesure où je conserve l'une ou l'autre des grandes conventions. Le roman est le genre qui me sollicite le plus et qui exige 
aussi le plus de disponibilité. J'écris encore de la poésie en prose, je cherche à faire entrer dans le roman les découvertes de cette poésie sans pour autant écrire un récit ou un roman poétique.

V. \& I. - Dans Transit, vous avez collaboré avec la compositrice Micheline Coulombe Saint-Marcoux.

F. Théoret - Micheline Coulombe Saint-Marcoux avait une conception tout à fait moderne et innovatrice de la musique. Elle avait beaucoup d'exigences dans sa démarche musicale. Elle alliait une grande sensibilité à une grande intelligence rationnelle. Il y a peu de femmes qui composent. Quand je l'ai rencontrée, je lui ai parlé de la voix dans l'écriture et des musiques que j'avais décrites. Il s'est trouvé que dans sa musique résolument moderne, elle avait souvent travaillé avec des textes poétiques pour faire en sorte que la voix devienne un instrument parmi les instruments. Nous en avons parlé. Probablement aussi pour rendre un aspect corporel à une musique de recherche constante. Nous n'en avons pas parlé, mais je le pense ainsi.

Transit m'a posé la question du sens. J'enviais la musique qui n'avait pas à s'en préoccuper. La grande différence entre la musique et l'écriture, c'est la sémantique. Les mots sont porteurs de sens. Il y a du sens partout en littérature, même lorsqu'on tente de l'évacuer. On lutte alors contre le sens.

\section{V. \& I. - Pourquoi cette préoccupation de la réalité dans vos écrits?}

F. Théoret - Peut-être parce que j'ai eu souvent l'impression de vivre ailleurs. Quand j'étais enfant, j'étais continuellement dans la lune. J'ai parlé de mes fabulations à propos du langage, elles étaient très grandes.

On nous enseigne «connais-toi toi-même». C'est juste, mais il faut compléter, on se connaît dans un rapport avec les autres et avec la réalité. «La vie est ailleurs» peut être une fabulation dangereuse quand on ne connaît pas la réalité. Dans mes fictions, je sais que je suis souvent obsédée par la question de la réalité, par ce qu'en dit la tradition, par ce qu'en disent les poètes et les philosophes. Je l'interroge au féminin, le rapport à la réalité. Dans Intérieurs, le rapport à la réalité de la matière est sûrement féminin, c'est une amie écrivaine qui m'en a parlé. Quand je pense à une réalité, je réfléchis également sur la raison et la déraison, le pouvoir et la liberté. La liberté s'acquiert bien plus qu'elle ne nous est donnée.

Je vais inscrire cette entrevue dans la canicule d'août 1988 par un exemple tiré de l'actualité: Félix Leclerc est mort cette semaine. Le journal le Monde lui rend hommage. Le journaliste écrit que nous sommes six millions de Québécois parlant presque français. De quelle réalité est-il question?

Vivre la réalité, connaître sa propre inscription dans le temps et dans l'espace suppose des solidarités. J'ai toujours désiré qu'il y ait des formes de solidarité. 
V. \& I. - Cette solidarité existe souvent entre femmes dans vos écrits. Mais jamais entre femme et homme. Est-ce parce que cela n'existe pas encore dans la société?

F. Théoret - La solidarité entre femmes, oui. Elle existe cette solidarité et elle change l'existence. Il faut que les femmes la désirent consciemment pour qu'elle existe. Maintenant, je reconnais à peu près immédiatement une femme solidaire.

Entre homme et femme, une forme instinctive d'attraction-répulsion joue presque toujours alors que la solidarité ne peut naître que d'une conscience. Cette conscience est à la fois intelligence et générosité. Il faut également que femme et homme se reconnaissent comme des égaux. Actuellement, pour beaucoup d'hommes, l'autonomie des femmes est perçue comme une catastrophe. Ils se vengent.

V. \& I. - Oui, mais entre certaines femmes, ça existe et ça propulse en avant.

F. Théoret - Certainement. Et je dois beaucoup à l'écriture des femmes. En même temps, je ne dois rien. Je tente d'en faire une parole plutôt qu'un discours lorsque j'aborde le rapport de solidarité. Autrement dit, la solidarité est vitale, je n'en fais pas une question idéologique. Je sais que la question idéologique est la grande question de ce siècle. J'ai beaucoup lu là-dessus.

V. \& I. - On dirait que depuis Nous parlerons comme on écrit, vous vous distancez un peu de ces problèmes énormes que sont les problèmes de la société actuelle, que vous retournez dans la mémoire vers votre jeunesse, vers des personnages de jeunes en tout cas... Et il me semble qu'en même temps, vous vous approchez d'une plus grande lisibilité dans l'écriture. Alors, pour en arriver à ma question, comment voyez-vous aujourd'hui l'apport de la modernité? Est-ce que vous avez laissé en arrière ou mis entre parenthèses pour un certain temps ces préccupations formalistes ou théoriques?

F. Théoret - J'essaie d'intégrer mes préoccupations théoriques. Mais je n'ai pas été formaliste, ou plutôt, j'avais trop de résistance face au formalisme, c'est pourquoi j'ai quitté la Barre du jour en 1969.

Aujourd'hui, l'apport de la modernité, je le vois dans une attitude face à l'écriture: il faut innover dans le langage.

La modernité a beaucoup cuvré autour de la question du sujet et à propos de la primauté du signifiant sur le signifié. Ces apports de la modernité me sont toujours essentiels et je ne les mets pas entre parenthèses.

La modernité à décloisonné les genres jusqu'à ne conserver que le texte. Une voix pour Odile est inscrit dans cette démarche du fragment. 
J'ai modifié mon langage du côté de la «lisibilité» (je n'ai jamais aimé ce terme) parce que je trouve essentiel de raconter. La phrase est souvent plus simple, mais l'ensemble du texte ne l'est pas. En outre, la psychanalyse n'a jamais eu beaucoup d'importance pour la modernité québécoise, elle en a dans mes réflexions.

Cela dit, quand on attaque la modernité, je me sens concernée. J'y vois une invitation à la régression.

V. \& I. - Peut-on dire que ce rapport qui vous préoccupe entre le moi et le collectif vous rapproche d'un certain réalisme - sans les prétentions, ou peutêtre la naïveté, de l'ancien réalisme? Dans la note qui précède votre récit «L'homme qui peignait Staline» dans la Nouvelle Barre du jour, par exemple, vous définissez l'enjeu du récit comme le rapport entre la langue et la pensée confronté au temps individuel et social. N'est-ce pas, dans une version qui intègre les connaissances de notre époque, ce qu'essayait de faire le réalisme traditionnel?

F. Théoret - J'aime le réalisme et je n'ai rien contre les grandes conventions du genre romanesque. Le roman permet l'invention d'une structure qui va se justifier par la cohérence du texte. En ce sens, la forme romanesque invite à l'invention. Et mes préoccupations dans l'écriture ne sont pas traditionnelles.

Le rapport entre le subjectif et le collectif me rapproche du réalisme très certainement. Je désire mettre en question la rationalité du roman réaliste. Ce qui me dérange dans le réalisme traditionnel, c'est l'apaisement qu'il procure en dépit des situations dramatiques qui en font la trame. Cet apaisement est lié à l'écriture du roman realiste traditionnel qui ne remet pas vraiment en question ce que parler veut dire dans un rapport social. Partant de là, je sais que ce que j'écris à propos de mes personnages féminins n'est pas excessif mais réaliste. Mes personnages féminins se sentent effrités par la réalité. Ils ne veulent plus être effrités par la réalité, alors mes personnages sont en parcours.

V. \& I. - Vous me rappelez l'image dans un de vos poèmes, d'une «flamme vive» en cette fin de siècle. On dirait que c'est cette flamme qui brûle au centre de votre parcours, de celui de vos personnages... Et l'amour qui est le point de transformation vers lequel ils sont en marche.

F. Théoret - Cet amour que vous nommez si bien, je l'associe au risque avec soi-même et à même ses propres ressources. L'amour est un peu comme la littérature. Pour qu'il y ait littérature, il faut la métamorphose du langage parlé. L'amour arrache le narcissisme de l'individu, il le métamorphose. À partir de soi, l'amour rend capable d'un geste gratuit et de générosité. L'amour suscite le désir d'un apport personnel à la collectivité. Ce sont des femmes et des hommes solidaires qui m'ont appris cela. 